\title{
A Phase Transition in the Water Coupled to a Local External Perturbation
}

\author{
Dmitri Volchenkov and Ricardo Lima \\ CNRS, Centre de Physique Theorique, Luminy Case 907, \\ 13288 Marseille Cedex 09, France \\ E-mailvolchen@cpt.univ-mrs.fr, lima@cpt.univ-mrs.fr
}

August 10, 2018

\begin{abstract}
A flux of ideal fluid coupled to perturbation is investigated by nonperturbative methods of the quantum field theory. Asymptotic behavior of the flux coupled to perturbation turns out to be similar to that of superfluids.
\end{abstract}

PACS numbers:03.40 G, 47.55

\section{Introduction}

The concept of spontaneous symmetry breaking is an important one in modern theoretical physics. It is known in statistical physics, condensed matter, and nuclear physics, as well as in relativistic field theory. An example of model subject to such a phase transition is given by an ideal fluid coupled to local external perturbation, [1]. For example, one may consider a pressure pulse localized in space of inviscid incompressible unbounded fluid which rises a fluid current with a net momentum flux $\mathcal{P}$. Then $\mathcal{P}$ plays the role of an order parameter, and the symmetry is spontaneously broken when $\mathcal{P} \neq 0$.

Statistical properties of the symmetrical phase of water response for the local external perturbation were discussed in [1] in details. As an example of such a behavior one imagines an eddy risen around a point wise distinct pressure perturbation. It was shown that the energy of hydrodynamic perturbation is confined within the eddy formed around the pulse region and demonstrates a power like decay $\sim r^{-4}$ outside this region, [1]. 
In the present paper we consider the water response in the case of symmetry broken, i.e., when $\mathcal{P} \neq 0$. Since the symmetry breaking does not affect the scaling properties of the theory, the universal quantities for the long-range asymptotic behavior of the correlation functions are the same as in the symmetrical phase. We demonstrate that if $\mathcal{P} \neq 0$ the leading order of long-range water response for perturbation is not determined by the scaling degrees of freedom, but by the anomalies risen due to an explicit symmetry breaking. The results achieved would give a key for better understanding of various aspects in the studies of the ocean surface. For example, one can consider the problem of generating of circulation motions (meanders) close to ocean currents. The grown up meanders is about to separate from the main current forming the stable closed rings (which averaged lifetime is about 2-3 years) of hundreds of kilometers in diameter drifting slowly along the main current (with an averaged speed of $2-10 \mathrm{~cm} / \mathrm{s}$ ), [3]- 4 .

The leading order of correlation functions stationary spectra in the fully developed turbulence theory of Kolmogorov [5] as well as in the statistical theory of waves based on the Zakharov's kinetic equations [3] can be found from pure phenomenology in principle. However, that is not the case for the water coupled to perturbation. The matter is of presence of redundant degrees of freedom connected to perturbation as well as to hydrodynamic equations themselves. To get rid of these degrees of freedom we propose a physically relevant hypothesis on the mechanism coupling water to perturbation.

In [1] we used the renormalization group ( $R G$ ) technique to justify such an additional assumption for the case of $O(3)$-symmetrical perturbation. In the framework of RG method the physical degrees of freedom are to be replaced by the scaling degrees which are related to the physical degrees through the RG transformations of fields and parameters of the theory. Since the properties of scaling degrees of freedom are governed by a group structure (the renormalization group) one investigates them much easier then those of the origin problem. The results obtained from RG-analysis are considered as somewhat statistical steady state limit of the physical system. The renormalized correlation functions are distinguished from their physical analogies only by normalization conditions, so that they can be also used for the analysis of asymptotic behavior of the physical system. The investigation of the relevant scaling degrees of freedom which has been brought about in [1] demonstrates that the asymptotic behavior $r>>l_{p}\left(l_{p}\right.$ is the perturbation scale) of water response can be determined unambiguously if one supposes a coupling between translation and rotation components of the velocity of fluid.

The stationary spectra can have place for those scale intervals which are transparent for currents of conserved quantities. The conserved energy current from the large-scale region of pumping into the small-scale range of viscous dissipation allows one to adjust 
the well known Kolmogorov's spectrum in the fully developed turbulence (the Five Thirds Law). However, the inertial range of Kolmogorov lies apart from the scale spectrum of our interest. The transparency interval related to the enstrophy current (the squared averaged vorticity) $\mathcal{E}$ is exactly the scale of the problem considered, though the enstrophy current gives us the spectrum of vorticial component of the velocity field only,

$$
\mathcal{E}^{1 / 2} k \simeq A(k),
$$

where $\mathbf{A}$ is the vector velocity potential:

$$
\mathbf{v}(\mathbf{x}, \mathbf{t})=-\operatorname{grad} \phi(x, t)+\operatorname{rot} \mathbf{A}(\mathbf{x}, \mathbf{t}),
$$

and $\phi$ is the scalar velocity potential. However, the spectrum for $\phi(k)$ is still unknown from these phenomenological considerations.

In language of the critical phenomena theory the spectrum (1) determines the critical dimension $\Delta[A]$ of the field $A, \Delta[A]=1$. The use of critical dimension allows to compute the spectrum of any correlation function of the field $A$ by simple dimensional counting. For example, for the pair correlation function in Fourier representation $D_{A}(k) \equiv\langle\mathbf{A}(\mathbf{k}) \mathbf{A}(-\mathbf{k})\rangle$, one has the asymptotics

$$
D_{A}(k) \sim k^{\Delta[A]}, \quad \Delta\left[D_{A}\right]=2 \Delta[A]-d,
$$

where $d$ is the dimension of space.

Further phenomenological considerations allow to determine the spectra of quantities which can be measured in experiments, for example, for the energy as a function of distance apart from the perturbation point, $E(r)$, one can obtain:

$$
\mathcal{E} r^{-4} \sim E(r) .
$$

These results were derived in [1] for the case of $O(3)$-symmetrical perturbation and justified within the framework of RG-approach. Formally, they are still valid for the case of symmetry broken, $\mathcal{P} \neq 0$.

The assumption on the mechanism coupling water to perturbation proposed in [1] allows to fix the spectrum for $\phi$ in the form

$$
\phi(k) \sim k^{7 / 12} .
$$

The confinement of energy within the region of $O(3)$-symmetrical perturbation could be interpreted as a kind of short-range order (in analogy with infinite ferromagnets), but the long-range order is suppressed. In case of symmetry broken spontaneously there is 
a nontrivial expectation value for $\langle\phi\rangle=\alpha(\mathbf{x})$ (in analogy to the arising of spontaneous magnetization in ferromagnets) engaged in long-range order with large-scale current motion.

In a regular way $\alpha(\mathbf{x})$ is to be determined from the equation of state

$$
\left(\alpha-\alpha_{0}\right)=f(j)
$$

with some function $f(j)$ calculating usually in the framework of Feynman graph expansion, [6]. However, the power-like asymptotic solution for (6) can be derived readily from phenomenology: considering $\mathcal{P}$ as a new dimensional parameter of the theory with symmetry broken, one obtains the spectrum $\alpha(k)$ in the form

$$
\alpha(k) \sim k .
$$

Continuing the analogy with ferromagnets, one can conclude that the ordered phase (of broken symmetry) water response can be described by a quantity analogous to the longitudinal susceptibility

$$
\chi_{L}=\frac{\partial \alpha}{\partial j},
$$

determined by

$$
\chi_{L} \equiv \int d \mathbf{x}\langle[\phi(\mathbf{x})-\alpha(\mathbf{x})][\phi(\mathbf{0})-\alpha(\mathbf{0})]\rangle .
$$

By the way, from the critical phenomena theory point of view the main problems of the theory in the non-symmetrical phase are to determine an explicit form for the function $f(j)$ in (6) and to justify the phenomenological result (7).

The plan of the paper follows: First, in the second section, for the convenience of readers we briefly reproduce the main result of [1] on model of ideal unbounded fluid coupled to perturbation concluding the section by a discussion on the explicit symmetry breaking. The statistical properties of hydrodynamical system can be described by a partition function of statistical mechanics with a classical euclidean action. As a result we derive the classical action functional designed to describe the long-range asymptotic behavior of the water coupled to perturbation. The relevant functional turns out to be analogous to that one of the abelian Higgs model well-known in relativistic field theory and superconductivity.

In the next section we observe the basic properties of the theory in case of an explicit $U(1)$-symmetry breaking and consider the physical consequences for the asymptotic behavior of the model. These properties are dramatically different from those demonstrated by the model in the symmetrical case discussed in [1]. In the latter phase scaling degrees of freedom were completely determined by the vector velocity potential $\mathbf{A}$, though in 
presence of comparably strong fluid flow the vector velocity components are confined in the flux and their contributions are irrelevant for the asymptotic behavior of the system. Looking for the stable stationary solutions, we obtain an infinite countable set of such solutions distinguished one from the other by energy gaps. In particular, a "ground state" solution can be interpreted as a pure laminar flow $(\mathbf{A} \equiv \mathbf{0})$.

In the fourth section we consider the asymptotic for $\alpha(r)$ in case of the laminar flow; it is provided by a Goldstone asymptotics arising by an explicit $U(1)$-symmetry breaking which has place in the real physical system.

To account the contributions of eddies into flux for "excited states" we construct an instanton solution for the theory of water coupled to perturbation and find the interval of validity for the phenomenological result (7) in the fifth section.

In the Section 6, we discuss the results obtained from the point of view of dynamical systems theory. We conclude in the last section.

\section{The Model of Ideal Unbounded Fluid Coupled to Perturbation}

In [1] it was shown that after elimination of all redundant degrees of freedom the problem considered possesses the entire $U(1)$-gauge symmetry [ [ 8 . In case of $j \neq 0$ this symmetry turns out to be hidden by an explicit symmetry breaking term appearing in the effective action functional. To reveal the hidden symmetry of hydrodynamics of ideal fluid we first suppose $j=0$. Then the equation of hydrodynamics takes the form

$$
\operatorname{div} \mathbf{v}(\mathbf{x}, \mathbf{t})=\mathbf{0}, \quad \mathbf{p}(\mathbf{x}, \mathbf{t})=\int \mathbf{d y} \frac{\partial_{\mathbf{i}} \mathbf{v}_{\mathbf{j}}(\mathbf{y}, \mathbf{t}) \partial_{\mathbf{j}} \mathbf{v}_{\mathbf{i}}(\mathbf{y}, \mathbf{t})}{|\mathbf{x}-\mathbf{y}|} .
$$

Here $\mathbf{v}(\mathbf{x}, \mathbf{t})$ is the velocity of fluid and $p(\mathbf{x}, \mathbf{t})$ is the pressure distribution. The trivial boundary conditions for the fields at infinity are implied.

The equations (10) do not lead to a hamiltonian in the usual way, since it is not possible to define the conjugated moments. Nevertheless, these equations can be derived from the classical Lagrangian $\mathcal{L}(\varphi)$ formulated in favor of the scalar and vector velocity potentials, [1]:

$$
\mathcal{L}\left(\phi, A_{i}, p\right)=\frac{1}{2} \int d \mathbf{x}\left[(\partial p)^{2}+(\partial \phi)^{2}+\frac{1}{2} F^{2}+p \partial_{i} v_{j} \partial_{j} v_{i}+J_{i} A_{i}\right],
$$

where we have introduced the eddy component of velocity field as a gauge invariant tensor $F^{k}=(\operatorname{rot} \mathbf{A})_{\mathbf{k}}=\partial_{\mathbf{i}} \mathbf{A}_{\mathbf{j}}-\partial_{\mathbf{j}} \mathbf{A}_{\mathbf{i}}, J_{i}=\partial_{j} F^{k}-\partial_{k} F^{j}(i \neq k \neq j)$ is the vorticity conserved for ideal fluid, and the tensor $v_{i} v_{j} \equiv\left(\partial_{i} \phi\right)\left(\partial_{j} \phi\right)+F^{i} F^{j}-\partial_{i} \phi F^{j}-\partial_{j} \phi F^{i}$ (the last two terms are vanished when integrated over $\mathbf{x}$ with trivial boundary conditions). 
With no coupling to pressure field $(p=0)$ (11) is invariant under the following transformations of fields

$$
\left\{\begin{array}{l}
A_{i}(\mathbf{x}) \rightarrow \mathbf{A}_{\mathbf{i}}(\mathbf{x})-\partial_{\mathbf{i}} \mathbf{\Lambda}(\mathbf{x}), \\
\phi(\mathbf{x}) \rightarrow \phi(\mathbf{x}) \mathbf{e}^{\mathrm{iu} \Lambda(\mathbf{x})}
\end{array}\right.
$$

where $u \Lambda(\mathbf{x})$ is an arbitrary continuous, differentiable phase function. The relations (12) express the $U(1)$-gauge symmetry of the functional (11) $(U(1)$ is the group of multiplication by complex numbers). In accordance to the Noether's theorem (12) relates to two conserved currents, vorticity, defined above,

$$
\partial_{i} J_{i}=0,
$$

and a current related to rotations in the complex plain $U(1)$ :

$$
I_{i}=\phi^{*} \partial_{i} \phi-\phi \partial_{i} \phi^{*}, \quad \partial_{i} I_{i}=0 .
$$

The statistical properties of mechanical system of an infinite number of degrees of freedom can be derived from the partition function of statistical mechanics $Z=\operatorname{Tr}\left(e^{-S}\right)$ with somewhat classical euclidean action functional,

$$
S\left(\phi, A_{i}, p\right)=\frac{1}{2} \int\left[(\partial p)^{2}+(\partial \phi)^{2}+\frac{1}{2} F^{2}+p \partial_{i} v_{j} \partial_{j} v_{i}\right]
$$

The last term in (15) does not meet the entire symmetry (12), since the pressure field as it is included in the action functional contains somewhat redundant degrees of freedom, we therefore can integrate it over in the partition function $Z$. The result of functional integration does not depend on $p(\mathbf{x}, \mathbf{t})$. This procedure is reduced to elimination of the quadratic term proportional to $(\partial p)^{2}$ from (15) and the replacement of the $U(1)$-symmetry breaking term by

$$
\frac{1}{2} \int d \mathbf{x} \partial_{i} v_{j}(\mathbf{x}, \mathbf{t}) \partial_{\mathbf{j}} \mathbf{v}_{\mathbf{i}}(\mathbf{x}, \mathbf{t}) \int_{\mathbf{V}_{\mathbf{p}}} \mathbf{d} \mathbf{y} \frac{\partial_{\mathbf{i}} \mathbf{v}_{\mathbf{j}}(\mathbf{y}, \mathbf{t}) \partial_{\mathbf{j}} \mathbf{v}_{\mathbf{i}}(\mathbf{y}, \mathbf{t})}{|\mathbf{x}-\mathbf{y}|},
$$

which relates the fluctuations of velocity fields risen by the perturbative pulse in the perturbed region $V_{p}$ to those fluctuations apart from $V_{p}$.

In [1] we investigated possible contributions of (16) into the action functional considering the insertions of various power like composite operators. It was shown that the only component which is important from the RG point of view have to be $U(1)$-gauge symmetrical, i.e.,

$$
m^{2} \phi^{2}
$$

in the first order, where $m^{2}$ is somewhat mass parameter (the coefficient of the relevant RG-invariant composite operator). The use of Ward identities which express the $U(1)$-gauge invariancy of the theory allows to demonstrate that all other combinations 
of quadratic operators are ultra-violet (UV) finite, i.e., the relevant correlation functions do not have UV-divergencies, and then they do not participate in scaling degrees of freedom.

Instantly close to the region of perturbation the pressure pulse rises the wave motions with eigenmodes $k>k_{0} \simeq V_{p}^{-1 / 3}$. Due to strong nonlinearity of interaction in hydrodynamical equations the eigenmodes of oscillations spread very fast from a band of order $V_{p}^{-1 / 3}$ over the whole spectrum, and various multipole oscillations of any type are arisen with time. Clearly, the long-range fluid behavior will depend to some extent on the statistical properties of wave mode coupling. Following [1] we suppose the simplest model for the coupling mechanism by inclusion of the $\varphi^{4}$-type interaction term into (15) with a wave modes coupling constant $g>0$. In accordance with the general critical phenomena approach we note that the accounting of highest oscillation harmonics, i.e., $\varphi^{6}, \varphi^{8}$, and so on cannot alter the large-distance asymptotic behavior of water response if $g \neq 0$. Again, since we are interested in $U(1)$-gauge symmetrical term the only amendments into action functional have to be of the form, $\sim g \phi^{4}$.

As a resulting hypothesis we obtain the effective action functional to be:

$$
S\left(\phi, A_{i}\right)=\frac{1}{2} \int d \mathbf{x}\left[(\partial \phi)^{2}+\frac{\mathbf{1}}{\mathbf{2}} \mathbf{F}^{2}+\mathbf{m}^{2} \phi^{2}+\frac{\mathbf{1}}{\mathbf{3}} \mathbf{g} \phi^{4}\right]
$$

which is designed to describe the asymptotic properties of water response.

The action (18) has some redundant degrees of freedom, the gauge degrees, with unknown dynamics. As a consequence it is not renormalizable, and it has no solutions in the massless limit $m^{2}=0$, [6]. To construct a renormalizable theory we introduce an abelian gauge geometrical structure:

(i) $\phi(\mathbf{x})$ and $\phi^{*}(\mathbf{x})$ are vectors for $U(1)$ transformations,

(ii) The derivative $\partial_{i}$ is replaced by the covariant derivative $\nabla_{i}$ :

$$
\nabla_{i}=\partial_{i}+i u_{0} A_{i}
$$

where $u_{0}$ is the coupling constant of interaction between the scalar and rotational components of the velocity potential $\varphi$ (analogous to the electron charge $e$ in electrodynamics).

(iii) It follows that the curvature tensor is $i u_{0} F_{i j}$ :

$$
i u_{0} F_{i j}=\left[\nabla_{i}, \nabla_{j}\right]=i u_{0}\left(\partial_{i} A_{j}-\partial_{j} A_{i}\right) .
$$

(iv) Since the $U(1)$-gauge group is abelian $(\mathbf{A}(\mathbf{x}, \mathbf{t})$ is a translation invariant), one can write the parallel transporter $U(C)$ along any continuous contour $C$ which is an element of $U(1)$. In terms of a line integral:

$$
U(C)=\exp \left[-i u_{0} \oint_{C} A_{i}(s) d s_{i}\right]
$$


as a consequence of vorticity conservation for ideal fluid. Thus, the rotational component of velocity potential just carries on the fluctuations of the scalar potential field $\phi(\mathbf{x})$. By the way, two solutions for different points $\phi(\mathbf{x}, t)$ and $\phi(\mathbf{y}, t)$ are related through the parallel transporter (20), where $C$ is an integration path connecting the points $\mathbf{x}$ and $\mathbf{y}$, [8].

Now, the gauge degrees of freedom (correspondent to invariancy of velocity with respect to the $\partial_{i} \Lambda$-shifts of vector potential $A_{i}$ ) can be taken into account by the usual procedure analogous to the Faddeev-Popov quantization [6]. In particular, it leads to inclusion of a gauge dependent term into the action functional,

$$
S\left(A_{i}, \phi\right)=\frac{1}{2} \int d \mathbf{x}\left[\left|\nabla_{\mathbf{i}} \phi\right|^{2}+\frac{\mathbf{1}}{\mathbf{2}} \mathbf{F}_{\mathbf{i j}}^{2}+\zeta^{-1}\left(\partial_{\mathbf{i}} \mathbf{A}_{\mathbf{i}}\right)^{2}+\mathbf{m}^{2} \phi^{2}+\frac{\mathbf{1}}{\mathbf{3}} \mathbf{g} \phi^{\mathbf{4}}\right],
$$

where $\zeta$ is an arbitrary valued $(\zeta \in[0, \infty))$ auxiliary gauge parameter of the theory. The model (21) demonstrates the existence of a statistically steady state independently of the details of velocity evolution.

In [1] the model (21) has been investigated in the symmetrical phase, $m^{2}>0$. The crucial distinction between symmetry implementation in the cases of positive and negative signs for $m^{2}$ lies though in the structure of the "ground state" (i.e., the expectation value of velocity potential).

Suppose, first, that $\mathbf{A}=\mathbf{0}$ in (21), then one has the standard model of a scalar unharmonic oscillator. For $m=0$ the oscillator is subject to a phase transition. At the classical level in the symmetrical phase $\left(m^{2}>0\right)$ the oscillator model describes the fluctuations having the trivial expectation value of the field, $\langle\phi\rangle=0$ (see Fig. 1.a). If $m^{2}<0$, the system allows an infinite number of possible expectation values related to each other by the unitary transformation group $U(1)$. In particular, if one fixes a phase parameter of the group $U(1)$ under certain physical conditions, then for the field $\phi$ there are two possible mean values (see Fig. 1.b)

$$
\alpha(\mathbf{x}) \equiv\langle\phi\rangle= \pm \sqrt{\mathbf{m}^{2} / \mathbf{g}}=\alpha_{\mathbf{0}}(\mathbf{x}) .
$$

The latter situation is usually referred to as spontaneously broken symmetry. Rise of a net fluid current from the region of initial perturbation into outside (see Fig.2) one can treat as a result of spontaneously symmetry breaking which can be described by (21) with $m^{2}<0$.

If we held $\mathbf{A}=\mathbf{0}$, the lagrangian is still invariant under the set of $U(1)$-transformations with no gauge section. When $j=0$, the Goldstone theorem predicts the appearance of a massless degree of freedom corresponding to unphysical "angular motion" for which there is no restoring force. The physical interpretation of such a degree of freedom would be 
the following: the quantity (8) diverges as $j \rightarrow 0$, i.e., an infinitely small initial fluid flow $j$ risen by perturbation generates the nontrivial expectation value for vector potential, $\alpha \neq 0$.

If we assume that $j \neq 0$, then the action (21) has the $U(1)$-symmetry breaking explicitly by the new term

$$
-\int d \mathbf{x} \mathbf{j}(\mathbf{x}) \operatorname{Re}[\phi(\mathbf{x}, \mathbf{t})]
$$

where $\operatorname{Re}[\phi(\mathbf{x}, \mathbf{t})]$ is the real part of the complex valued field $\phi$. This symmetry breaking term gives in the first order in $j$ a mass proportional to $j^{1 / 2} \partial_{i} I_{i}$ (the axial current $I_{i}$ is no more conserved) to the unphysical angular degree of freedom correspondent to rotations in the complex plain (i.e., (8) has no more divergent).

The situation is though to be changed dramatically if one includes the vorticial velocity component into consideration $(\mathbf{A} \neq \mathbf{0})$. Due to so called Higgs mechanism the angular degree of freedom does not produce divergences in (8) even in the zero order in $j$, and the gauge field $A_{i}$ acquires a mass without spoiling the gauge invariance and renormalizability of the theory, [8]-[9]. These ideas which are quite familiar in the weak interactions theory and superconductivity allow an heuristic interpretation also in hydrodynamics: In the symmetrical phase $(j=0)$ A plays the purely transporting role for scalar velocity potential fluctuations from one point to another in accordance with (20). When the symmetry is broken spontaneously, A acquires the longitudinal polarization degree of freedom giving it a mass; as a direct consequence a vector potential field can penetrate only exponentially into the fluid flow with a range proportional to the inverse of the acquired mass. Like a superconductor expels a magnetic field from its interior, except for a thin layer at the surface over which the field decreases exponentially, the fluid flow ousts the eddies from its interior onto the periphery. The microscopic origin of the Higgs phenomenon in hydrodynamics lies in screening currents of fluid compensating the external velocity rotational component (see Fig.3).

In [1] it was shown that the scaling degrees of freedom proportional to $g$ are vanished from the asymptotic behavior of fluid involved into eddy motion. By the way, considering $j=0$ (say, on the periphery skin of a large-scale current), one can omit the term $g \phi^{4}$ from (21) to obtain the relevant effective action functional. However, if $j \neq 0$ (within the current), from the heuristic point of view it is obvious that the statistical steady state should be free of coupling to the vector potential $\mathbf{A}$, and the solution for $\alpha(\mathbf{x})$ (i.e., (7)) is to be determined by a Goldstone asymptotics, [11. 


\section{The Gauge-Invariant $U(1)$ Theory of the Water Cou- pled to Perturbation}

In the present section we develop the heuristic ideas of the preceding one. We derive the action functional for the theory in case of symmetry spontaneously broken and investigate its properties.

Implementing the local transformation

$$
\left\{\begin{array}{c}
\phi(\mathbf{x}) \rightarrow[\alpha(\mathbf{x})+\phi(\mathbf{x})] \mathrm{e}^{\mathbf{i} \mathbf{u} \mathbf{\Lambda}(\mathbf{x}) / \alpha(\mathbf{x})} \\
A_{i}(\mathbf{x}) \rightarrow \mathbf{A}_{\mathbf{i}}(\mathbf{x})-\frac{\mathbf{u}}{\alpha(\mathbf{x})} \partial_{\mathbf{i}} \mathbf{\Lambda}(\mathbf{x})
\end{array}\right.
$$

to the model action functional relevant to the system of equations (10), we fix the gauge in such a way that

$$
u \Lambda(\mathbf{x})=\mathbf{2} \pi \mathbf{n} \alpha(\mathbf{x}), \quad \mathbf{n} \in \mathbf{Z} .
$$

The parameter $u$ which characterize the coupling strength of vorticial and translational velocity components in this gauge is related to a circulation, $\Gamma$,

$$
\Gamma_{n} \equiv \oint \mathbf{A} \mathbf{d x}=\frac{2 \mathbf{n} \pi}{\mathbf{u}}
$$

Physical degrees of freedom are become clear now (since $\Lambda(\mathbf{x})$ is gauged away from the theory):

$$
\begin{aligned}
S\left(A_{i}, \phi\right)= & \frac{1}{2} \int d x\left[\left|\nabla_{i} \phi\right|^{2}+\frac{1}{2} F_{i j}^{2}+\zeta^{-1}\left(\partial_{i} A_{i}+\sqrt{2} u \alpha \cdot \operatorname{Im}[\phi]\right)^{2}+m_{\phi}^{2} \phi^{2}+\right. \\
& \left.+m_{A}^{2} A^{2}+\frac{1}{3} g \phi^{4}+u_{1}^{2} \phi A^{2}+\frac{4}{3} g_{1} \phi^{3}-j \phi+S(\alpha)\right],
\end{aligned}
$$

where we have denoted $u_{1}^{2}=u^{2} \alpha, g_{1}=g \alpha, m_{A}^{2} \equiv 2 \alpha u, m_{\phi}^{2}=2 g \alpha^{2}-m^{2}$, and $\operatorname{Im}[\phi]$ is an imaginary part of $\phi$. Comparing (27) and (21), one can see that the vector field A obtains the longitudinal polarization degree of freedom for which is expressed in (27) as the new mass term $m_{A}^{2} A^{2}$. The longitudinal components of the vector fields $A_{i}$ and $\operatorname{Im}[\phi]$ are ghosts, which both cancel against the Faddeev-Popov ghost [12] all having the same $\operatorname{mass} m_{A}$.

The behavior of (27) is in a way very different from (21): the unphysical imaginary part of the scalar velocity potential $\phi$ disappears and the vector field A obtains a mass so that the vorticial velocity component is short-range only, i.e., it is repelled completely from the flux.

The standard way to illustrate the last sentence is to demonstrate that the response of the flux for an elementary vortex immersed in is equal to zero [14. Consider the constant shift transformation (a purely vorticial constant velocity component)

$$
F_{i j} \rightarrow F_{i j}+f_{i j}
$$


for the gauge section $F_{i j}$ in the disordered theory (21). Then the partition function $Z\left[f_{i j}\right]$ is invariant under gauge transformations:

$$
f_{i j} \rightarrow f_{i j}+\partial_{i} \lambda_{j}-\partial_{j} \lambda_{i},
$$

since it can be compensated by the appropriate change $A_{i} \rightarrow A_{i}+\lambda_{i}$. Therefore, in a gauge-invariant theory (21) one has

$$
Z\left[F_{i j}+f_{i j}\right]=Z\left[F_{i j}\right]
$$

furthermore, one notes that the constant $f_{i j}$ can be removed from (21) by the transformation:

$$
A_{i} \rightarrow A_{i}+x_{j} f_{i j} .
$$

In the phase with long-range correlations (21), the change (31) is equivalent to somewhat change of trivial boundary conditions for the equations (10) at infinity. In particular, this yields the new term into the partition function

$$
Z\left[f_{i j}\right]-Z[0]=\frac{\varepsilon_{A}}{u^{2}} \int d \mathbf{x} \mathbf{f}_{\mathbf{i j}}^{2},
$$

where $\varepsilon_{A}$ (the Lagrange multiplier) would be some function which has a natural interpretation as an amplitude of the response of the flux for an elementary vortex. Obviously, if the circulation $\Gamma<\Gamma_{0}$, where $\Gamma_{0}$ is some critical value correspondent to the phase transition point, the vector potential becomes short-range correlated, and the partition function $Z$ should not depend on $f_{i j}$. Thus $\varepsilon_{A}=0$ in the theory (27).

In case of the vector velocity potential $\mathbf{A}$ is strong enough then, because of circulation conservation, it can be allowed in the fluid flow in the form of narrow flux tubes. The relation (26) in this context means that there are always an integer number $n$ of such vorticial tubes in the flow, i.e., that each of them have a source and a sink (see Fig.4).

Varying (27) with respect to $A_{i}$ and $\phi^{*} \phi$ with the boundary condition (26) (i.e., fixing the circulation $u^{-1}$ to be constant in ideal fluid), one can easily estimate the energy of a flux with length $l_{f}$ as

$$
E \sim u^{2} m_{A}^{2} l_{f},
$$

which demonstrates the property inherent to a confinement phenomenon. Note, that (33) could be derived rigorously by considering of a Wilson's loop operator for a point-wise vorticial current,

$$
\mathcal{J}_{i}(\mathbf{x})=-\mathbf{i u} \oint \delta(\mathbf{x}-\mathbf{y}) \mathbf{d y},
$$

for (27) (see, for example [7]). 
This situation is analogous to that of superconductors [13]: if electrically charged bosons (Cooper's bound state of an electron pair) Bose-condensed then there the electric fields become short-range, and the magnetic fields are ousted from the interior. If finally a magnetic field is admitted inside a superconductor it can only come in some multiple vortices, never spread out because of Meissner effect. Following the analogy with superconductors, one can say that the source and the sink vortices confined in the fluid flow are kept together in a potential well, and the potential is being linearly proportional to their separation.

As it well known, [9]-[10], the hidden symmetry begets the hidden renormalizability: the divergence structure of renormalizable theory (21) is unaffected by spontaneous symmetry breaking, and the counterterms needed in (27) remain those of the symmetrical theory (21). Consequently, the critical dimensions calculated in [1] for the quantities in (21) are still valid formally also for (27).

\section{Goldstone Asymptotics of the Water Flux Cou- pled to Perturbation}

In the present section we construct (6) explicitly and justify the phenomenological result (7). We shall consider the theory in the ordered phase assuming that the velocity field has no vorticial components (i.e., the flux contains no vorticial pairs, $n=0$ in (25)). Therefore, to describe the statistical properties of the system we can integrate the partition function $Z[\phi, \mathbf{A}]$ over $\mathbf{A}$ eliminating the vector field $\mathbf{A}$ from the theory. The resulting partition function will depend solely on the scalar velocity potential, $Z[\phi]$, and the relevant action functional will be identical to those of scalar $\phi^{4}$-theory in the ordered phase (nonlinear $\sigma$-model):

$$
S=-\frac{1}{2} \int d \mathbf{x}\left[(\partial \phi)^{2}+\tau \phi^{2}+\frac{\mathbf{g}}{3 !}\left(\phi^{2}\right)^{2}-\mathbf{j} \boldsymbol{R e}[\phi]\right] .
$$

The distinguishing feature of (35) is the presence of Goldstone singularities which arise due to an explicit $U(1)$-symmetry breaking. The physical origin of these singularities is following, [14]: the scalar velocity potentials with different orientation in the complex plane, however, correspond to the same fluid velocity and though to the same energy. The relevant conserved current meets the Ward identity in the momentum representation:

$$
k_{i}\left\langle I_{i}(k) \phi(-k)\right\rangle=\langle\phi(0)\rangle .
$$

Taking $k \rightarrow 0$, one concludes that $\left\langle I_{i}(k) \phi(-k)\right\rangle$ must be singular in this limit:

$$
\left\langle I_{i}(k) \phi(-k)\right\rangle_{k \rightarrow 0}=\langle\phi(0)\rangle \frac{k_{i}}{k^{2}}+\ldots
$$


The general solution for Goldstone asymptotics in (35) was given in [11] for the unbounded theory and then generalized in [15] to the theory in a half-space. In particular, the hypothesis [16] was proven in [11] for any order of $\epsilon$-expansion $(2 \epsilon=4-d)$ with $j, k \rightarrow 0$ : the equation of state (6) has the form

$$
\left(\alpha-\alpha_{0}\right)=a j^{1-\epsilon}+b j+\ldots,
$$

and the longitudinal susceptibility (8) is to be

$$
\chi_{L}=a_{1} j^{-\epsilon}+b_{1}+\ldots,
$$

the numerical coefficients $a, b, a_{1}, b_{1}$ are specified in [11]. For the transversal susceptibility, $\chi_{T} \sim j^{-1}$ as it follows from the Goldstone theorem. Following the discussion in [17], formulae (38) and (39) can be interpreted as a Goldstone scaling (by analogy to critical scaling) for which $j$ and $k \sim 1 / r$ play the role of significant parameters. The certain Goldstone dimensions $\Delta^{G}$ belong to $k, j$, and $\left.\alpha(\mathbf{x})\right|_{\mathbf{j}=\mathbf{0}}$ :

$$
\Delta^{G}[k]=1, \quad \Delta^{G}[j]=2, \quad \Delta^{G}[\alpha]=d-2 .
$$

In contrast with critical dimensions (40) are known precisely as well as the normalized scaling functions of the simplest correlation functions [17]. The last relation in (40) justifies the result (7): performing the inverse Fourier transformation, one obtains at three dimensions

$$
\alpha(r) \sim \frac{1}{r^{2}} .
$$

\section{The Instanton Solutions for the Theory of Water Coupled to Perturbation}

In the previous sections we have considered the stationary (with no time dependence) stable solutions of (27) which correspond to the saddle points (solutions of the hydrodynamical equations). However, in the case discussed, $j \neq 0$, the actual hydrodynamical equations, posses the non-constant solutions also.

In the previous sections we have shown that there is a countable set of possible stable stationary solutions (enumerated by an integer number $n$ ) for the system of flux coupled to perturbation distinguished one from the other by the energy gaps (33). Obviously, the non-constant statistically steady solutions are related to a specific mechanism of gap generation (the generation of new pairs of eddies in the flux), i.e., they describe possible transitions between constant solutions with different $\Gamma_{n}$ (26). 
Another interpretation can be used: since the source and sink eddies are confined together in the potential well in the fluid flux, one can consider a tunneling process of the eddy pair into another potential well. This tunneling process can be provided by an instanton solution [14]. The contribution of instantons into the correspondent partition functional $Z$ is indeed irrelevant if we are interested in relatively short periods of time $t<t_{0}$, where $t_{0}$ is a "tunnelling time". However, for $t>t_{0}$, it becomes very large.

Consider the action (35) in case of the symmetry broken spontaneously. Classical minima of this action defined from the equation:

$$
\Delta \phi_{a}-m^{2} \phi_{a}+\frac{g}{2}\left(\sum_{1}^{2} \phi_{b}^{2}\right) \phi_{a}=j
$$

where $\phi_{1,2}$ are the real and imaginary parts of the field $\phi, \phi=\left(\phi_{1}+\phi_{2}\right) / \sqrt{2}$. We use the anzatz

$$
\phi=\mu(r) e^{i \frac{\Lambda}{\alpha}},
$$

which gives the equation for $\mu(r)$ in the form,

$$
\mu^{\prime \prime}-m^{2} \mu-\frac{2}{r^{2}} \mu+g \mu^{3}=j .
$$

There exists a solution to (44) with the properties:

$$
\mu(r \rightarrow 0) \rightarrow 0, \quad \mu(r \rightarrow \infty) \rightarrow \alpha_{0} .
$$

The problem of existence and stability of the solution of (44) with (45) were discussed in [14]. The effective asymptotical solution is given in the preceding section by the Goldstone asymptotics. Suppose now that one has introduced a set of vortices, placed at the points $\mathbf{x}_{\mathbf{a}}$ with circulations $\Gamma_{a}$ into the flux (35). The partition function of statistical mechanics $Z$ is then to be presented in the form (in case of $\alpha_{0} \gg u^{2}$ )

$$
Z=Z_{0} Z_{\text {inst }}
$$

where $Z_{0}$ is the standard partition function of the theory (35) and

$$
Z_{\text {inst }}=\operatorname{Tr}\left(\exp \left[\frac{\alpha_{0}}{2 u^{2}} \int d \mathbf{x} \sum_{a \neq b} \frac{2 \pi \Gamma_{a} \Gamma_{b} L}{\left|\mathbf{x}_{\mathbf{a}}-\mathbf{x}_{\mathbf{b}}\right|}+C \sum_{a} \Gamma_{a}^{2}\right]\right)
$$

( $L$ being the size of the flux pattern considered; the second term is the vortex selfenergy). One can see that in case of large fluid flux $\alpha_{0}$ and, consequently, strong confining property the vortices revolving alternatively are combined into pairs. Such pairs have very small influence on the correlation functions and are irrelevant in case of large $\alpha_{0}$. The asymptotics provided by the instanton solutions is just the same as (41). 


\section{Discussion from the Point of View of Dynamical Systems Theory}

The Navier-Stockes equation for an ideal fluid can be replaced by the relation for the pressure field, and the Galilean invariance of hydrodynamical equations is manifested as a $U(1)$-gauge invariance (12).

We shall concern with the phase space (of infinite dimensionality) relevant to the dynamical system of water coupled to perturbation and limit ourselves to a qualitative consideration. Picturing the instant states of the system in the phase space, we obtain its phase diagram. The stable stationary solutions discussed in [1] and in the present paper can be interpreted as the attraction regions or fixed points of trajectories of the system in the phase space.

Consider the manifold of initial conditions correspondent to the only solution of disordered phase $(\mathcal{P}=0)$. In [1] we have shown that it can be realized exceptionally as an eddy risen around a point-wise distinct perturbation. One can imagine this manifold as a torus covered by the trajectories tending to some stable cycle (see Fig.5).

If we chose a point apart from the torus as the initial condition (for example, the points $A$ or $B$ on the diagram Fig. 5), the system will leave the vicinity of the torus and tends to some region of attraction which is closed in a sense that there are no trajectories going out of it. This behavior represents a phase transition in the language of statistical theory. Within the attraction region the trajectory passes consequently through an infinite set of fixed points distinguishing by the $\Gamma_{n}$ values (12). Most of these points are hetreoclinic, and so that they are unstable in a sense that the smallest deviation from the certain set of initial conditions will make the system trajectory to jump to some other point. These processes, in principle, are to be described by the instanton solutions (see Sec. 5).

This technique would provide us with information on the transition probabilities between the particular heteroclinic fixed points. Such a quantity could be naturally interpreted within the context of the dynamical systems theory. Let us surround each heteroclinic fixed point by a ball of radius $\varepsilon$ and consider a fixed point $n_{0}$ which corresponds to the solution with $\Gamma_{n_{0}}$ (see Fig. 5). Taking $\varepsilon$ to be small enough, we can make the volume of each ball to be finite. Denote the volume of a ball sector starting from which the trajectory of the system drops into the $\varepsilon$-vicinity of the other point, $n_{i}$, as $V_{\varepsilon}\left(n_{0} \rightarrow n_{i}\right)$. Then, one can introduce the quantity

$$
P\left(n_{0} \rightarrow n_{i}\right)=\frac{V_{\varepsilon}\left(n_{0} \rightarrow n_{i}\right)}{\sum_{k, k \neq 0} V_{\varepsilon}\left(n_{0} \rightarrow n_{k}\right)}
$$

which is analogous to a transition probability defined in the statistical theory. If the 
point which we have chosen is a homoclinic attractive fixed point, the probability (48) then tends to zero. $0<P<1$ for heteroclinic points, and $P=1$ for a repelling point. We do not know a priori whether there are some homoclinic attractive fixed points in the region of attraction (see Fig. 5) or there are only the heteroclinic points. We expect though that in case of $\alpha \gg u$ there is a degeneracy of solutions in a sense that they are predicted by the Goldstone asymptotics.

\section{Conclusion}

In a conclusion one can say that the flux of ideal fluid coupled to local external perturbation in the region $r>l_{p}$ demonstrates asymptotically some properties similar to those of superfluids. In [1] and in the present paper we have considered the statistically steady asymptotic solutions of the model by various nonperturbative techniques of the quantum field theory. The results on RG-analysis, Goldstone asymptotics, and instanton-type solutions are, by the way, exact, and they demonstrate that the long-standing hydrodynamical problem of water coupled to perturbation, in principle, can be treated as a critical phenomenon.

The relevant physical system contains too many redundant degrees of freedom. To fix the statistically stable behavior in the system one needs to add some extra assumptions on the character of perturbation as well as on the character of wave modes coupling. The model describing such a behavior is subject to a phase transition managed basically by the perturbation symmetry. Roughly speaking, the symmetry properties of the initial perturbation define whether the vorticial or translational fluid velocity components is the most important one for the long-range asymptotic fluid response.

\section{References}

[1] Preprint CPT-98/P3712, D. Volchenkov, R. Lima, Critical Behavior of the Water Coupled to a Local External Perturbation

[2] V. P. Krasitskii, J. Fluid Mech. 272, 1-20 (1994)

[3] A.S. Monin, V.P. Krasitskii Phenomena on the Ocean Surface, Gidrometeoizdat, St.-Peterburg, 1985 (in Russian)

[4] Kamenkovich V.M., Koshlyakov M.N., Monin A.S. Synoptic Eddies in Oceans, Gidrometeoizdat, St.-Peterburg, 1982. (in Russian) 
[5] A.S. Monin, A.M. Yaglom, Statistical Fluid Mechanics (MIT Press, Cambridge, Mass., 1975), Vol. 2.

[6] E. Brezin, D.J. Wallace, K.G. Wilson, Phys. Rev. B, 7, 1, 232 (1973)

[7] J. Zinn-Justin Quantum Field Theory and Critical Phenomena (Clarendon, Oxford, 1990)

[8] M. Guirdy, Gauge Field Theories (J. Wiley \& Sons, NY, 1991)

[9] E. Leader, E. Predazzi An Introduction to gauge theory and modern particle physics (Cambridge, 1996)

[10] S. Coleman, in Laws of Hadronic Matter (ed. by A. Zichichi; Academic Press, 1975)

[11] Nalimov M. Yu., Theor. and Math. Phys., 80, 2, 212 (1989).

[12] B.S. de Witt, Phys. Rev. 162, p. 1195-1239 (1967)

[13] G.'t Hooft, Vol. 19 in Advanced Series in Mathematical Physics, World Scientific, 1994.

[14] A. M. Polyakov Vol 3, Gauge Fields and Strings in Contemporary Concepts in Physics, Harwood Acad. Publ., 1987.

[15] M. Yu. Nalimov, Theor. and Math. Phys., 102, 2, 163 (1995)

[16] A. Z. Patashinsky, V. L. Pokrovsky, JTPH 64, 4, 1445 (1973)

[17] Vasil'ev A.N. Functional Methods in the Quantum Field Theory and Statphysics (to be published) (in Russian) (1998) 


\section{CAPTIONS FOR FIGURES}

FIGURE 1.

a.) At the classical level in the symmetrical phase $\left(m^{2}>0\right)$ the oscillator model describes the fluctuations having the trivial expectation value of the field, $\langle\phi\rangle=0$.

b.) If $m^{2}<0$, the system allows an infinite number of possible expectation values related to each other by the unitary transformation group $U(1)$. In particular, if one fixes a phase parameter of the group $U(1)$ under certain physical conditions, then for the field $\phi$ there are two possible mean values.

\section{FIGURE 2.}

Rise of a net fluid current from the region of initial perturbation into outside.

\section{FIGURE 3.}

To a Higgs phenomenon in hydrodynamics. When the symmetry is broken spontaneously, A acquires the longitudinal polarization degree of freedom giving it a mass $m_{A}$. The fluid flow ousts the eddies from its interior onto the periphery.

\section{FIGURE 4.}

The kink-type solutions for fluid flow. The source and the sink vortices confined in the fluid flow are kept together in a potential well, and the potential is being linearly proportional to their separation.

\section{FIGURE 5 .}

a) The manifold of initial conditions correspondent to the only solution of disordered phase $(\mathcal{P}=0)$. If we chose a point apart from the torus as an initial condition (for example, the points $A$ or $B$ ), the system will leave the vicinity of the torus and tends to some region of attraction which is closed in a sense that there are no trajectories going out of it. This tendency represents a phase transition in a language of statistical theory.

b) Surround each heteroclinic fixed point by a ball of radius $\varepsilon$ and consider a fixed point $n_{0}$ which corresponds to the solution with $\Gamma_{n_{0}}$. 